\title{
One4All Cooperative Media Access Strategy in Infrastructure Based Distributed Wireless Networks
}

\author{
Zhang, Qi; Fitzek, Frank H.P.; Iversen, Villy Bæk
}

Published in:

IEEE Wireless Communications and Networking Conference

Link to article, DOI:

10.1109/WCNC.2008.269

Publication date:

2008

Document Version

Publisher's PDF, also known as Version of record

Link back to DTU Orbit

Citation (APA):

Zhang, Q., Fitzek, F. H. P., \& Iversen, V. B. (2008). One4All Cooperative Media Access Strategy in Infrastructure Based Distributed Wireless Networks. In IEEE Wireless Communications and Networking Conference (pp. 15011506). IEEE. https://doi.org/10.1109/WCNC.2008.269

\section{General rights}

Copyright and moral rights for the publications made accessible in the public portal are retained by the authors and/or other copyright owners and it is a condition of accessing publications that users recognise and abide by the legal requirements associated with these rights.

- Users may download and print one copy of any publication from the public portal for the purpose of private study or research.

- You may not further distribute the material or use it for any profit-making activity or commercial gain

- You may freely distribute the URL identifying the publication in the public portal 


\section{One4All Cooperative Media Access Strategy in Infrastructure Based Distributed Wireless Networks}

\author{
Qi Zhang \\ Department of Communication, \\ Optics \& Materials \\ Technical University of Denmark \\ Lyngby, Denmark \\ Email: qz@com.dtu.dk
}

\author{
Frank H.P. Fitzek \\ Department of Communications \\ Technology \\ Aalborg University \\ Aalborg, Denmark \\ Email: ff@es.aau.dk
}

\author{
Villy B. Iversen \\ Department of Communication, \\ Optics \& Materials \\ Technical University of Denmark \\ Lyngby, Denmark \\ Email:vbi@com.dtu.dk
}

\begin{abstract}
In this paper we propose the one4all cooperative access strategy to introduce a more efficient media access strategy for wireless networks. The one4all scheme is designed for the infrastructure based distributed wireless network architecture. The basic idea is that mobile devices can form a cooperative cluster using their short-range air interface and one device contends the channel for all the devices within the cluster. This strategy reduces the number of mobile devices involved in the collision process for the wireless medium resulting in larger throughput, smaller access delay, and less energy consumption. Based on an analytical model, the proposed strategy is compared with the two existing strategies RTS/CTS (request to send/ clear to send) and packet aggregation. The results show that the proposed cooperative scheme has similar throughput performance as packet aggregation and it has much higher throughput than the conventional RTS/CTS scheme. Furthermore, the newly introduced cooperative scheme outperforms packet aggregation in terms of channel access delay and energy consumption.
\end{abstract}

\section{INTRODUCTION}

Current wireless local area networks (WLANs) suffer from an inefficient wireless access mechanism. For example, the IEEE 802.11 WLAN standard product can provide up to 54Mbps transmission rate at the physical layer. The recent IEEE $802.11 \mathrm{n}$ proposals aim at providing physical layer transmission rates up to $600 \mathrm{Mbps}$. But theoretical throughput limits exist [13], [15] due to the medium access control (MAC) and the physical layer (PHY) overhead, the backoff time in case of contention, the inter-frame space (IFSs) and others. Therefore, to achieve high throughput values at the network layer, research should focus not only on higher physical layer data rates but also on more efficient MAC strategies to reduce the aforementioned overhead. So far the most popular and effective strategy to enhance WLAN throughput is packet aggregation [6]-[9], [12]. But packet aggregation has inevitable drawbacks, for instance the throughput gain is highly dependent on the arrival traffic pattern. Packet aggregation does improve the throughput for bursty traffic such as FTP, but it would not improve throughput so much for non-bursty traffic such as VoIP. Furthermore, it also may cause longer channel access time which in turns leads to higher energy consumption and unfair channel usage between mobile devices.

In this paper, we propose a new cooperative media access strategy named one4all. It is designed for infrastructure based distributed wireless network architecture. The basic idea of the proposed strategy is that every mobile device within a cooperative cluster does not contend for the channels for itself anymore, but benefit from cooperation among its neighboring mobile devices.

In this paper we will not address RTS/CTS based CSMA/CA (carrier sense multiple access with collision avoidance) scheme but focus on explaining packet aggregation and the one4all strategy. Then we will derive the throughput, channel access delay and energy consumption performance of packet aggregation and one4all strategy. The performance comparison of these three strategies will be given at the end.

\section{CSMA/CA based Packet Aggregation Strategy}

Packet aggregation is a popular strategy to improve throughput in wireless networks based on CSMA/CA. It has been addressed in many research works [6]-[9], [12] There are two main categories of packet aggregation [1], [3], [5], [10], [11], [14] depending on aggregation on different sub-layers: Aggregation of multiple MAC Protocol Data Units (A-MPDU) and aggregation of multiple MAC Service Data Units (AMSDU). The idea of A-MPDU is to concatenate multiple MAC PDUs into a single PHY SDU (PSDU). MAC PDUs can be concatenated if they are available and have the same physical source and destination address. The length of concatenation should not exceed a given threshold. The idea of A-MSDU is to combine multiple MAC SDUs with the same MAC addresses from a higher layer into a big MAC PDU. The detailed description of these two aggregation schemes can be seen in proposals from TGnSync or WWiSE [1], [11]. We use the A-MPDU strategy as a representative packet aggregation method here. Additionally, block ACK is also used in the A-MPDU strategy. The A-MPDU frame structure diagram is shown in Fig. 1.

\section{ThE One4all STRATEGY}

Packet aggregation has three drawbacks which will be analyzed in detail in Section IV: (i) The achievable throughput depends on the arrival traffic pattern. Packet aggregation is good for bursty traffic but not for smooth traffic. (ii) Long channel access delay in packet aggregation might lead to unfair 


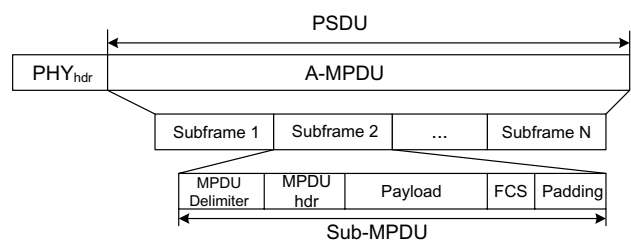

Fig. 1. Frame structure in A-MPDU packet aggregation scheme

media usage between mobile devices if delay sensitive service such as VoIP does not have higher priority. (iii) Longer channel access delay also causes higher energy consumption in the mobile device. To overcome these drawbacks, we propose the one4all strategy for CSMA/CA.

The proposed strategy aims at infrastructure based distributed wireless network architecture. The mobile devices in the network are assumed to have two air-interfaces: one for the link between AP (access point) and mobile devices referred to as access link (AL) and the other for the short-range link among mobile devices (SRL). The mobile device is capable to form a cluster with the mobile devices in its proximity by the short-range link. The idea of one4all strategy is that the mobile device cooperates with the other devices in its clusters and only one device in a cluster contends to access the channel instead of all of them contending to access the channel. The contending device also receives the block ACK and distributes the block ACK over the short-range link. The advantage of the proposed strategy is that: first the collision probability of the transmitted frames by the contending mobile devices is reduced and second the remaining devices in a cluster can access the channel free of contention. The contention duty and transmission sequence in the cluster can be maintained by a logical token ring topology like [16]. The signalling between the devices in a cluster is exchanged over the short-range link.

The proposed strategy also has its drawback since the achievable throughput gain depends on the number of the cooperative mobile devices in one cluster. But it can be integrated with packet aggregation strategy to exploit the advantages of both strategies. Fig. 2 shows the integrating of the onetall scheme and packet aggregation.

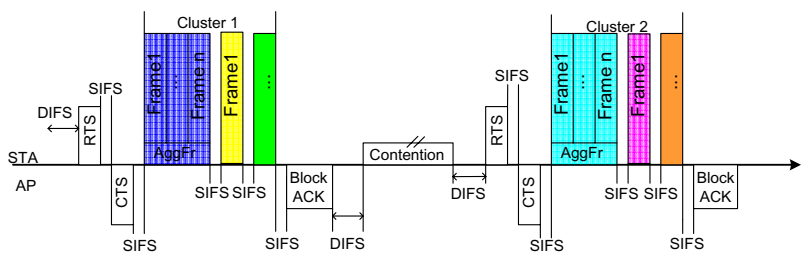

Fig. 2. Contention and transmission procedure in one4all scheme

\section{Performance Analysis}

In this section we will derive and analyze the saturation throughput, channel access delay, energy consumption performances of packet aggregation and one4all strategy. The
TABLE I

NOTATION LIST

\begin{tabular}{|c|c|}
\hline Notation & Meaning \\
\hline$n$ & the number of contending mobile devices \\
\hline$W$ & min (initial) contention window \\
\hline$m$ & backoff stage $\left(W_{\max }=2^{m} W\right)$ \\
\hline$\sigma$ & one unit slot time \\
\hline$\delta$ & propagation delay \\
\hline sifs & short interframe space \\
\hline disf & distributed interframe space \\
\hline$R$ & channel data rate \\
\hline$T_{\text {int }}$ & the time interval between two consecutive transmissions \\
\hline$P_{s}$ & the probability that a transmission is successful \\
\hline$T_{s}$ & trans time of a single successful frame transmission \\
\hline$T_{c}$ & the average trans time of transmission with collision \\
\hline$T_{o}$ & $\begin{array}{l}\text { the time that a mobile device has to wait when its frame } \\
\text { transmission collides, before sensing the channel again. }\end{array}$ \\
\hline$\Psi / \bar{\Psi}$ & $\begin{array}{l}\text { The number of consecutive idle slot times before a } \\
\text { transmission takes place/ expectation of } \Psi\end{array}$ \\
\hline$N_{c} / \overline{N_{c}}$ & $\begin{array}{l}\text { the number of collisions of a frame until its successful } \\
\text { transmission/ expectation of } N_{c}\end{array}$ \\
\hline$D_{b} / \overline{D_{b}}$ & the backoff delay/ expectation of $D_{b}$ \\
\hline$C_{b k} / \overline{C_{b k}}$ & the value of the backoff counter/ expectation of $C_{b k}$ \\
\hline$N_{f r} / \overline{N_{f r}}$ & $\begin{array}{l}\text { the number of times that a counter freezes before a } \\
\text { counter reaching zero/expectation of } N_{f r}\end{array}$ \\
\hline$T_{p} / \overline{T_{p}}$ & $\begin{array}{l}\text { the time used for successful transmission of a payload/ } \\
\text { expectation of } T_{p}\end{array}$ \\
\hline$D_{c} / \overline{D_{c}}$ & the channel access delay/ expectation of $D_{c}$ \\
\hline$S_{r t s} / T_{r t s}$ & size/ transmission time of a RTS frame \\
\hline$S_{c t s} / T_{c t s}$ & size/ transmission time of a CTS frame \\
\hline$S_{p} / T_{p} / \overline{T_{p}}$ & $\begin{array}{l}\text { size/ transmission time of a PDU payload/ expectation of } \\
T_{p}\end{array}$ \\
\hline$S_{p h y}^{h} / T_{p h y}^{h}$ & size/ transmission time of a PHY header \\
\hline$S_{m a c}^{h} / T_{m a c}^{h}$ & size/ transmission time of a MAC header \\
\hline$S_{a c k} / T_{a c k}$ & size/ transmission time of a block ACK frame \\
\hline
\end{tabular}

derivation is based on the analytical model in [2], [17] which derived the saturation throughput and backoff delay of basic CSMA/CA and RTS/CTS strategy. we extend the derivation of the existing analytical model for a system using new strategies, furthermore energy consumption is addressed.

In [17] it is assumed that the network consists of $n$ contending mobile devices and each device has an arrival packet for transmission immediately after its completion of a successful packet transmission, a kind of saturation case. The notation are listed in Table I.

\section{A. Throughput Analysis}

For the model presented in [2], [17], it is assumed that each transmission is a renewal process, no matter whether it is successful or not. Therefore, the saturation throughput $\eta$ can be calculated according to the payload transmitted during a single renewal interval between two consecutive transmissions. The expression of saturation throughput $\eta$ of RTS/CTS based CSMA/CA is given in [17] as following:

$$
\eta=\frac{\overline{T_{p}}}{\overline{T_{\text {int }}}}=\frac{P_{s} \overline{T_{p}}}{\bar{\Psi} \sigma+P_{s} T_{s}+\left(1-P_{s}\right) T_{c}}
$$

where, how to derive $\Psi, P_{s}, T_{c}$ refers to [17].

The throughput of a system with packet aggregation $\eta^{a}$ can 
similarly expressed as

$$
\eta^{a}=\frac{P_{s} \overline{N_{a} T_{p}}}{\bar{\Psi} \sigma+P_{s} T_{s}^{a}+\left(1-P_{s}\right) T_{c}}
$$

Where, $\overline{N_{a}}$, the average number of packets in an aggregated frame, and $T_{s}^{a}$, the transmission time of an aggregated frame transmission.

Based on the frame structure of A-MPDU (Fig. 1), the average transmission time of an aggregated frame transmission $T_{s}^{a}$ is a function of $\overline{N_{a}}$. Hence, according to the packet aggregation MAC mechanism (see Fig. 3), $T_{s}^{a}$ can be calculated by

$$
\begin{aligned}
T_{s}^{a}= & T_{r t s}+\delta+\operatorname{sifs}+T_{c t s}+\delta+\operatorname{sifs}+T_{p h y}^{h}+ \\
& \overline{N_{a}}\left(T_{m d}+T_{m a c}^{h}+T_{p}\right)+\delta+\operatorname{sifs}+T_{a c k}+\delta+\operatorname{difs}
\end{aligned}
$$

So the key issue is to solve $\overline{N_{a}}$ which depends on the number of available packets in the buffer when a mobile device just finishes transmitting an aggregated frame. Packet aggregation is performed under the condition that the packets are available in the buffer and the number of packets in one aggregated frame must be smaller than a threshold, which means the number of packets in one aggregated frame is variable. Furthermore, to calculate the saturation throughput, the arrival traffic should meet the condition that there is at least one arrival packet in the buffer immediately after the mobile device completes an aggregated frame transmission. We generate such arrival traffic by a poisson process with additional constraints. The arrival rate of this poisson process has to meet the constraint that the probability of no arrival packet during time $T_{a c}$ is close to zero, denoted by $\varepsilon$ (e.g., $\varepsilon=10^{-4}$ ). $T_{a c}$ is the duration of the previous aggregated frame starting to contend until its successful completion transmission. Under this condition, it can be regarded as that there is at least one packet in the buffer.

Hence, the assumed poisson arrival process $P(i, t)$ with arrival rate $\lambda$ with constraints is expressed by

$$
P\left(0, T_{a c}\right)=e^{-\lambda T_{a c}}=\varepsilon
$$

then, there is $\lambda T_{a c}=-\ln \varepsilon$

The time $T_{a c}$ based on its definition can be given by

$$
T_{a c}=\overline{D_{c}}+T_{s}^{a}
$$

where, $\overline{D_{c}}$ is the channel access delay which will be discussed in the next subsection.

Based on the packet aggregation scheme, the average number of the packets in an aggregated frame is calculated according to basic expectation calculation with additional constraints that the packets in one frame can not exceed the threshold $\widehat{N}_{a}$. $\overline{N_{a}}$ is given by

$$
\begin{aligned}
\overline{N_{a}} & =\sum_{i=1}^{\widehat{N_{a}}} i \frac{\left(\lambda T_{a c}\right)^{i}}{i !} e^{-\lambda T_{a c}}+\sum_{i=\widehat{N_{a}}+1}^{\infty} \widehat{N_{a}} \frac{\left(\lambda T_{a c}\right)^{i}}{i !} e^{-\lambda T_{a c}} \\
& =\varepsilon\left(\sum_{i=1}^{\widehat{N_{a}}} i \frac{(-\ln \varepsilon)^{i}}{i !}+\sum_{i=\widehat{N_{a}}+1}^{\infty} \widehat{N_{a}} \frac{(-\ln \varepsilon)^{i}}{i !}\right)
\end{aligned}
$$

From Eq. 2, it is also clear that the achieved throughput gain depends on $\overline{N_{a}}$ which is a function of $\widehat{N_{a}}$ and the arrival rate of poisson process. If the arrival rate is low, $\overline{N_{a}}$ becomes small. Then the packet aggregation can not enhance the throughput that much.

To calculate throughput of one4all strategy $\eta^{c}$, first we define one contention and transmission period as the duration that the representative mobile device starts to contend until the available packets in the clusters being transmitted. In the period each mobile device sends one packet and mobile device also has one packet available in the buffer immediately after the period. So the throughput is expressed by

$$
\eta^{c}=\frac{c_{m} \overline{T_{p}} P_{s}}{\bar{\Psi} \sigma+P_{s}^{c} T_{s}^{c}+\left(1-P_{s}^{c}\right) T_{c}}
$$

where, $c_{m}$ is the number of mobile devices in a cluster, $P_{s}^{c}$ is the probability of a success transmission with one4all strategy and $T_{s}^{c}$ is the transmission time of a cooperative cluster.

To obtain $P_{s}^{c}$, we follows the same derivation given in [17], besides substituting $n$ the total number of contending mobile devices by $n / c_{m}$ the number of clusters, i.e., the number of representative contending mobile devices. Here, each cluster is assumed to have same number of mobile devices.

According to Fig. 2, the $T_{s}^{c}$ is calculated by

$$
\begin{aligned}
T_{s}^{c}= & T_{r t s}+\delta+\operatorname{sifs}+T_{c t s}+\delta+\operatorname{sifs}+c_{m}\left(T_{p h y}^{h}+T_{m a c}^{h}+T_{p}\right)+ \\
& \delta+\operatorname{sifs}+T_{a c k}+\delta+\operatorname{difs}
\end{aligned}
$$

\section{B. Energy Consumption Analysis \& Channel Access Delay}

The energy consumption depends on the energy consumed in different communication phases of the mobile device and the time that the mobile device staying at the corresponding state. There are four different possible states that a device can stay: transmission state, reception state, listening state and idle state. Their corresponding power consumptions are denoted by $P_{t x}, P_{r x}, P_{l i}, P_{i}$, respectively.

The mobile device is at the transmission state only when it sends RTS messages or data frames. The device is in reception state when it receives CTS messages or ACK messages from the access point or receives RTS message from other mobile devices. During DIFS and SIFS the device is in listening state. We assume that the mobile device performs a smart energy saving strategy, i.e., the mobile device can switch to the idle state when it adjusts its network allocation vector (NAV).

According to the packet aggregation strategy, the power level changes as shown in Fig. 3.

Based on the Fig. 3, the energy consumption of each packet is calculated by summing up all the energy consumption during such one contention and transmission period; then the sum is averaged by the number of packets in one aggregated frame. The contention and transmission period is defined as the time from the mobile device starting contention until it finally transmitting the frame successfully. Hence, the energy consumption for each packet can be expressed by

$$
E^{a}=\frac{1}{\overline{N_{a}}}\left(P_{t x} T_{t x}^{a}+P_{r x} T_{r x}^{a}+P_{l i} T_{l i}^{a}+P_{i} T_{i}^{a}\right)
$$




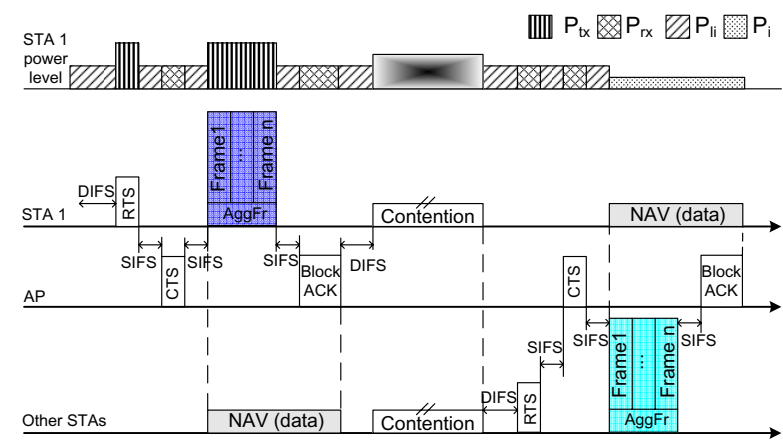

Fig. 3. The power level changing of mobile device 1 diagram

It is obvious that the sum of $T_{t x}^{a}, T_{r x}^{a}, T_{l i}^{a}$ and $T_{i}^{a}$ is equal to one contention and transmission period. It is composed of: the channel access delay $D_{c}$ and frame transmission time $T_{s}^{a}$.

Channel access delay $D_{c}$ is defined as the time duration from a mobile device starting to contend the channel until the instant that it can start to transmit the packet successfully. The average channel access delay can be given as

$$
\overline{D_{c}}=\overline{N_{c}}\left(\overline{D_{b}}+T_{c}+T_{o}\right)+\overline{D_{b}}
$$

The explanation of the expression is as following: 1. Before a mobile device can transmit packet successfully, it has collided with others for $\overline{N_{c}}$ times. 2. Before each contention try, it has waited for time $\overline{D_{b}} .3$ If there is a collision, it knows after time $T_{c}$. 4. After a collision occurs, it has to wait for time $T_{o}$ before sensing the channel again. $\overline{D_{b}}, T_{c}$ and $T_{o}$ are given as following [17]

$$
\begin{aligned}
\overline{D_{b}} & =\overline{C_{b k}} \sigma+\overline{N_{f r}}\left(P_{s} T_{s}^{a}+\left(1-P_{s}\right) T_{c}\right) \\
T_{c} & =T_{r t s}+\delta+\operatorname{difs} \\
T_{o} & =\text { sifs }+C T S_{\text {timeout }}
\end{aligned}
$$

The backoff delay $D_{b}$ depends on the product of $C_{b k}$ (the value of the backoff counter) and the slot time, and the backoff counter freezing duration. The average of the backoff counter is a random variable depending on the initial contention window and the backoff stages. The total duration of the backoff counter in freezing state depends on the number of times that the mobile device freezes the counter and the duration of each freezing.

To calculate $T_{t x}^{a}, T_{r x}^{a}, T_{l i}^{a}$ and $T_{i}^{a}$, we can sort the different state durations within $D_{c}$ and $T_{s}^{a}$ referring to Fig. 3, individually.

First $T_{t x}^{a}$ consists of two parts: $T_{t x, 1}^{a}$, the successful transmission time which includes transmission time of RTS, physical header and the PSDU with $\overline{N_{a}}$ subframes; and $T_{t x, 2}^{a}$, the time that the transmitted RTS collides which is the product of the average collisions and the transmission time of RTS.

$$
T_{t x, 1}^{a}=T_{r t s}+T_{p h y}^{h}+\overline{N_{a}}\left(T_{m d}+T_{m a c}^{h}+T_{p}\right) ; \quad T_{t x, 2}^{a}=\overline{N_{c}} T_{r t s}
$$

$T_{r x}^{a}$ also consists of two parts: $T_{r x, 1}^{a}$, the time that the mobile device receives other's RTS\&CTS during backoff duration, and
$T_{r x, 2}^{a}$, the mobile device receives its own CTS and ACK when its RTS is successfully transmitted.

$$
\begin{aligned}
& T_{r x, 1}^{a}=\left(\overline{N_{c}}+1\right) \overline{N_{f r}}\left(P_{s}\left(T_{r t s}+T_{c t s}\right)+\left(1-P_{s}\right) T_{r t s}\right) \\
& T_{r x, 2}^{a}=T_{c t s}+T_{a c k}
\end{aligned}
$$

$T_{l i}^{a}$ consists of three parts: the listening time during all backoff durations within channel access delay, the listening time when the transmitted RTC collides, and the listening time during a successful transmission.

$$
T_{l i}^{a}=\left(\overline{N_{c}}+1\right) T_{l i}^{b k}+\overline{N_{c}}(\delta+\operatorname{difs})+(3 \delta+3 \text { sifs }+ \text { difs })
$$

where, $T_{l i}^{b k}$ is the mobile device's listening time during a backoff duration. It includes the time spending on backoff counter and the listening duration when the counter freezes. $T_{l i}^{b k}$ is given by

$$
T_{l i}^{b k}=\overline{C_{b k}} \sigma+\overline{N_{f r}}\left(P_{s}(2 \delta+2 \text { sifs }+ \text { difs })+\left(1-P_{s}\right)(\delta+\operatorname{difs})\right)
$$

$T_{i}^{a}$ includes two parts: all the NAV time duration in the channel access delay and the idle time because of $T_{o}$.

$$
T_{i}^{a}=\left(\overline{N_{c}}+1\right) \overline{N_{f r}} P_{s} T_{n a v}+\overline{N_{c}} T_{o}
$$

where $T_{n a v}$ is one NAV data duration (see Fig. 3):

$$
T_{\text {nav }}=T_{p h y}^{h}+\overline{N_{a}}\left(T_{m d}+T_{m a c}^{h}+T_{p}\right)+\delta+\operatorname{sifs}+T_{a c k}+\delta
$$

For one 4all strategy, we only consider the energy consumption of the access link, because the power consumption of the short-range link is much lower, furthermore, the signalling on the short-range link is very little. To calculate the energy consumption of one 4all strategy, the difference is that when one representative mobile device contends the channel (i.e., sending RTS, receiving CTS, listening the channel states and backoff. etc.), the other mobile devices in the cluster are all in idle state. After the representative has caught the channel successfully, the remaining mobile devices alternately wake up to transmit their own packets. They switch to idle mode right away after completion of transmission. So the average energy consumption per packet is calculated by summing up all the energy consumption of the mobile devices in one cluster in one contention and transmission period; and then the sum is averaged by the number of mobile devices in one cluster. Note, we assume each mobile device can only have one packet to send each time the cluster catches the channel. Hence, the energy consumption for each packet can be given by

$$
E^{c}=\frac{1}{c_{m}}\left(P_{t x} T_{t x}^{c}+P_{r x} T_{r x}^{c}+P_{l i} T_{l i}^{c}+P_{i} T_{i}^{c}\right)
$$

The derivation of $T_{t x}^{c}, T_{r x}^{c}, T_{l i}^{c}$ is similar as those in packet aggregation strategy. $T_{t x}^{c}$ consists of $T_{t x, 1}^{c}$ all the successful transmission time and $T_{t x, 2}^{c}$ the time that the transmitted RTS collides;

$$
T_{t x, 1}^{c}=T_{r t s}+c_{m}\left(T_{p h y}^{h}+T_{m a c}^{h}+T_{p}\right) ; \quad T_{t x, 2}^{c}=\overline{N_{c}} T_{r t s}
$$

The expression of $T_{r x}^{c}$ and $T_{l i}^{c}$ is same as $T_{r x}^{a}$ and $T_{l i}^{a}$ just needing to replace the item $P_{s}$ in $T_{r x}^{a}$ and $T_{l i}^{a}$ by $P_{s}^{c}$. 
$T_{i}^{c}$ is the idle time of all the mobile devices in a cluster during one contention and transmission period. The contending mobile device has different idle time than the other devices in a cluster. So we calculate them separately.

The idle time of a contending device, $T_{i}^{c n d}$, consists of: $T_{i, 1}^{c n d}$, the total NAV time due to the transmission of other clusters; $T_{i, 2}^{c n d}$, all the $T_{o}$ time because of its transmitted RTSs collision; and $T_{i, 3}^{c n d}$, its idle time during all the transmission time of the other devices in its cluster.

$T_{i, 1}^{c n d}=\left(\overline{N_{c}}+1\right) \overline{N_{f r}} P_{s}^{c}\left(c_{m}\left(T_{p h y}^{h}+T_{m a c}^{h}+T_{p}+\delta+\operatorname{sifs}\right)+T_{a c k}+\delta\right)$ $T_{i, 2}^{c n d}=\overline{N_{c}} T_{o}$

$T_{i, 3}^{c n d}=\left(c_{m}-1\right)\left(T_{p h y}^{h}+T_{m a c}^{h}+T_{p}\right)+c_{m}(\operatorname{sifs}+\delta)$

The idle time of one non-contending device, $T_{i, 0}^{\text {nocnd }}$, consists of the transmission time of the other mobile devices within the cluster and channel access delay ${ }^{1}$ during which the contending device is contending the channel.

$T_{i, 0}^{\text {nocnd }}=\left(\left(c_{m}-1\right)\left(T_{p h y}^{h}+T_{m a c}^{h}+T_{p}\right)+c_{m}(\operatorname{sifs}+\delta)+T_{a c k}\right)+\overline{D_{c}}$

Hence, the total idle time of all the non-contending mobile devices in a cluster $T_{i}^{\text {nocnd }}$ can be given as

$$
T_{i}^{\text {nocnd }}=\left(c_{m}-1\right) T_{i, 0}^{\text {nocnd }}
$$

In summary $T_{t x}^{c}$ includes the time of the representative sending RTSs, its own packet and the time of the remaining mobile devices sending their packets. $T_{r x}^{c}$ includes the time of the representative receiving CTS and block ACK for the cluster, the RTSs and CTSs of the other clusters. $T_{l i}^{c}$ is the time of the representative listening the channel. $T_{i}^{c}$ is the sum of the time that all mobile devices are in idle states during one contention and transmission period. Fig. 4 makes it easy to understand the way to calculate $T_{i}^{c}$.

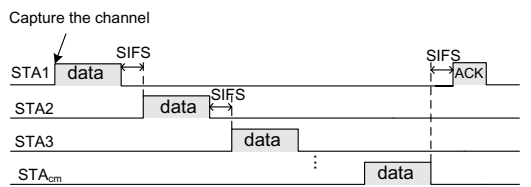

Fig. 4. Active/idle switching diagram of the mobile devices in one cluster

\section{NuMERICAL RESUltS}

To illustrate the proposed cooperative access strategy outperforming other existing MAC strategies, some numerical results are presented in this section. The assumption of the parameters is listed in Table $\mathrm{II}^{2}$.

In this section we compare the performance of three different MAC strategies (i.e., the one4all strategy, packet aggregation strategy and conventional RTS/CTS strategy) in CSMA/CA. We focus on the performance of throughput, channel access delay and energy consumption. It should be

\footnotetext{
${ }^{1}$ Note: the channel access delay $\overline{D_{c}}$ in Eq. 13 can be calculated by Eq. 10 just needing to replace the $P_{s}$ by $P_{s}^{c}$.

${ }^{2}$ In the table $S_{m a c}^{h}$ incl. FCS, $S_{r t s}$ incl. $S_{p h y}^{h}, S_{c t s}$ incl. $S_{p h y}^{h}$
}

TABLE II

ASSUMED PARAMETER VALUE LIST

\begin{tabular}{|l|l|l||l|l|l|}
\hline Notation & Value & Unit & Notation & Value & Unit \\
\hline$W$ & 32 & & sifs & 10 & us \\
$m$ & 3 & & difs & 50 & us \\
$n$ & {$[4-60]$} & & $\delta$ & 1 & us \\
$S_{p}$ & 1023 & byte & $\widehat{N_{a}}$ & 4 & \\
$S_{m a c}^{h}$ & 34 & bytes & $c_{m}$ & 4 & \\
$S_{p h y}^{h}$ & 16 & bytes & $\sigma$ & 20 & us \\
$S_{r t s}^{h}$ & 20 & bytes & $R$ & 11,54 & Mbps \\
$S_{c t s}$ & 14 & bytes & $P_{t x}$ & 2 & $\mathrm{~W}$ \\
$T_{p}$ & $P / R$ & us & $P_{r x}$ & 0.9 & $\mathrm{~W}$ \\
$\ldots$ & $\ldots$ & $\ldots$ & $P_{l i}$ & 0.9 & $\mathrm{~W}$ \\
$T_{c t s}$ & $S_{c t s} / R$ & us & $P_{i}$ & 0.04 & $\mathrm{~W}$ \\
\hline
\end{tabular}

mentioned here that these three strategies are not completely independent. For instance one4all cooperative access strategy and packet aggregation strategy are both built on RTS/CTS scheme. More generically, cooperative access strategy can also be built on top of packet aggregation scheme, but in the example they are implemented independently.

The throughput comparison Fig. 5 shows that both of packet aggregation and one4all strategies can greatly enhance the throughput. The reason lies in that they save SIFS or DIFS time between each transmission of a mobile device. The difference between them is that one4all reduces the collision probability and saves the backoff time. On the other hand, in one4all strategy each frame carries its own PHY header but in packet aggregation multiple MAC frames can use the same PHY header, which makes packet aggregation more efficient at this aspect. So throughput comparison results are impacted by all these elements. In this example, the one4all strategy slightly outperforms packet aggregation. The throughput result matches with the conclusion in [17] that based on RTS/CTS scheme, the saturation throughput is insensitive to the number of the mobile devices in the network.

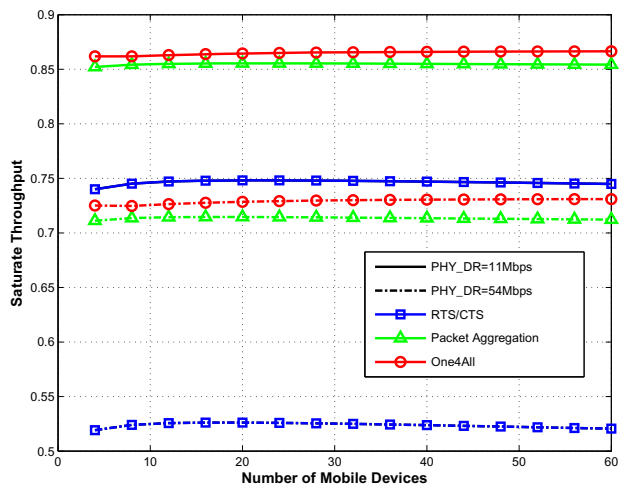

Fig. 5. Throughput comparison of different MAC strategies in CSMA/CA

As for the channel access delay performance, we define the average per packet channel access delay, which is the calculated channel access delay averaged by the number of MAC PDUs (PHY PDUs) the mobile device(s) transmitting after the channel is obtained.

Fig. 6 shows the average per packet channel access delay 
comparison. It shows that the average per packet channel access delay of packet aggregation scheme and RTS/CTS scheme is much longer than one4all scheme. Furthermore, the channel access delay increases with an increasing number of devices. The longer channel access delay has great impact on fairness of media usage between different mobile devices, if they have different types applications. It might also affect the QoS of the delay sensitive services such as VoIP, if there is no priority differentiation between applications.

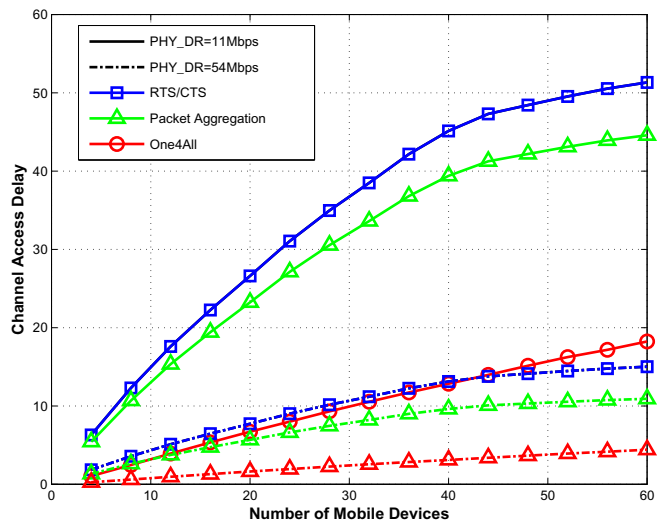

Fig. 6. Average per packet channel access delay comparison

The energy consumption performance comparison of the three strategies is shown in Fig. 7. Because the conventional RTS/CTS and packet aggregation strategies have much longer channel access delay, more energy is wasted in the channel contention duration, even though we have assumed that both of these two schemes have smart energy saving scheme. The energy comparison clearly shows that in case the number of mobile devices in the network exceeds 30, the conventional RTS/CTS consumes as over three times energy as the one4all scheme does. Packet aggregation strategy uses approximately as double the energy as the one4all cooperative scheme.

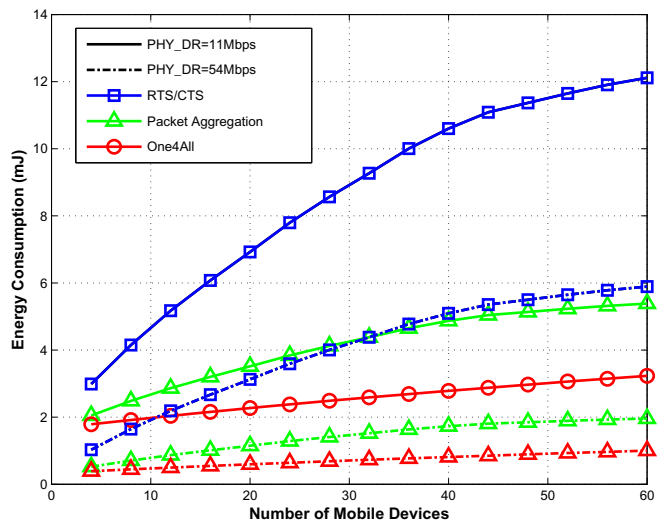

Fig. 7. Average energy consumption for one packet successful transmission

\section{CONClusions}

We propose one4all strategy for MAC protocol design for infrastructure based distributed wireless network, e.g. WLANs.
The proposed scheme significantly enhances the throughput compared with conventional RTS/CTS scheme. It overcomes the drawback of packet aggregation where the throughput performance is sensitive to the arrival rate of traffic. Namely, one4all strategy is suitable for both bursty and non-bursty traffic. Furthermore, it outperforms the packet aggregation scheme in terms of channel access delay and energy consumption. The proposed scheme can also be built on top of the packet aggregation scheme to enhance the throughput. The one4all strategy is a good illustration underlining the great potential of cooperation among mobile devices to solve the low uplink throughput issue in contention based wireless network.

\section{REFERENCES}

[1] IEEE 802.11n WWiSE. Wwise proposal: High throughput extension to the 802.11 standard. Technical report, Jan 2005.

[2] G. Bianchi. Ieee 802.11-saturation throughput analysis. IEEE Соттиnications Letters, 2(12):318-320, 1998.

[3] Enhanced Wireless Consortium. Ht mac specification. Technical report, Jan 2006.

[4] Frank H.P. Fitzek and Marcos D.Katz, editors. Cooperation in Wireless Networks: Principle and Applications. ISBN-10 1-4020-4710-X. 2006.

[5] Matthew S. Gast. 802.11 Wireless Networks: The Definitive Guide. ISBN-10: 0596100523. O'Reilly Media,, 2 edition edition, April 25, 2005.

[6] Seongkwan Kim, Youngsoo Kim, Sunghyun Choi, Kyunghun Jang, and Jin-Bong Chang. A high-throughput mac strategy for next-generation wlans. World of Wireless Mobile and Multimedia Networks, 2005. WoWMoM 2005. Sixth IEEE International Symposium on a, pages 278$285,2005$.

[7] Youngsoo Kim, Sunghyun Choi, Kyunghun Jang, and Hyosun Hwang. Throughput enhancement of ieee 802.11 wlan via frame aggregation. Vehicular Technology Conference, 2004. VTC2004-Fall. 2004 IEEE 60th, 4:3030-3034, 2004.

[8] Dzmitry Kliazovich and Fabrizio Granelli. On packet concatenation with qos support for wireless local area networks. ICC 2005 - 2005 IEEE International Conference on Communications and IEEE International Conference on Communications, 2:1395-1399, 2005.

[9] Tianji Li, Qiang Ni, D. Malone, D. Leith, Yang Xiao, and T. Turletti. A new mac scheme for very high-speed wlans. World of Wireless, Mobile and Multimedia Networks, 2006. WoWMoM 2006. International Symposium on a, page 10 pp., 2006.

[10] Yuxia Lin and Vincent W. S. Wong. Frame aggregation and optimal frame size adaptation for ieee $802.11 \mathrm{n}$ wlans. In GlobeCOM, San Francisco, Nov 2006

[11] IEEE $802.11 \mathrm{n}$ TGn Sync. Tgn sync proposal technical specification. Technical report, May 2005.

[12] Y. Nagai, A. Fujimura, Y. Shirokura, Y. Isota, F. Ishizu, H. Nakase, S. Kameda, H. Oguma, and K. Tsubouchi. 324mbps wlan equipment with mac frame aggregation. Personal, Indoor and Mobile Radio Communications, 2006 IEEE 17th International Symposium on, pages $1-5,2006$.

[13] Shao-Cheng Wang and Ahmed Helmy. Performance limits and analysis of contention-based ieee 802.11 mac. In 31st IEEE Conference on Local Computer Networks (LCN), Tampa, Florida, U.S.A., November 2006.

[14] Yang Xiao. Efficient mac strategies for the ieee 802.11n wireless lans. Wireless Communications and Mobile Computing, 6(4):453-466, 2006.

[15] Yang Xiao and J. Rosdahl. Throughput and delay limits of ieee 802.11. IEEE Communications Letters, 6(8):355-357, 2002.

[16] Qi Zhang, Frank H.P. Fitzek, and Villy B. Iversen. Design and performance evaluation of cooperative retransmission scheme for reliable multicast services in cellular controlled $\mathrm{p} 2 \mathrm{p}$ networks. In The 18th Annual IEEE International Symposium on Personal, Indoor and Mobile Radio Communications (PIMRC07), September 2007.

[17] Eustathia Ziouva and Theodore Antonakopoulos. Csma/ca performance under high traffic conditions: throughput and delay analysis. Computer Communications, 25(3):313-321, 2002. 